\title{
Low-level control software for the WEAVE spectrograph
}

Bernardo Salasnich, Carlos Martín Perez, José Miguel Delgado, Sergio Picó, Diego Cano Infantes, et al.

Bernardo Salasnich, Carlos Martín Perez, José Miguel Delgado, Sergio Picó, Diego Cano Infantes, Remko Stuik, Andrea Baruffolo, Gavin Dalton, Scott Trager, José Alfonso López Aguerri, Piercarlo Bonifacio, Antonella Vallenari, Esperanza Carrasco, Don Carlos Abrams, Kevin Middleton, "Lowlevel control software for the WEAVE spectrograph," Proc. SPIE 10707, Software and Cyberinfrastructure for Astronomy V, 107071X (6 July 2018); doi: $10.1117 / 12.2311469$

Event: SPIE Astronomical Telescopes + Instrumentation, 2018, Austin, Texas, United States 


\title{
Low-Level Control Software for the WEAVE Spectrograph
}

\author{
Bernardo Salasnich*a, Carlos Martín Pérez ${ }^{\mathrm{b}}$, José Miguel Delgado ${ }^{\mathrm{c}}$, Sergio Picó ${ }^{\mathrm{b}}$, Diego Cano

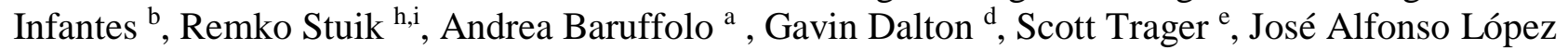 \\ Aguerri $^{\text {c }}$, Piercarlo Bonifacio ${ }^{\mathrm{f}}$, Antonella Vallenari ${ }^{\mathrm{a}}$, Esperanza Carrasco ${ }^{\mathrm{g}}$, Don Carlos Abrams ${ }^{\mathrm{b}}$, \\ Kevin Middleton ${ }^{\mathrm{d}}$ and the WEAVE Instrument Consortium. \\ ${ }^{a}$ Osservatorio Astronomico di Padova, INAF, Vicolo Osservatorio 5, 35122, Padova, Italy; ${ }^{\text {b Isaac }}$ \\ Newton Group, 38700 Santa Cruz de La Palma, Spain; 'Instituto de Astrofisica de Canarias, 38200 \\ La Laguna, Tenerife, Spain; ${ }^{d}$ RALSpace, STFC Rutherford Appleton Laboratory, OX11 0QX, UK; \\ ${ }^{e}$ Kapteyn Institut, Rijksuniversiteit Groningen, Postbus 800, NL-9700 AV Groningen, Netherlands;

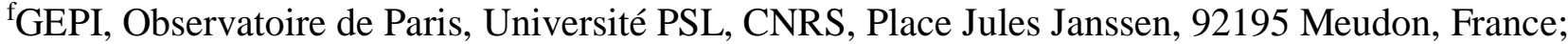

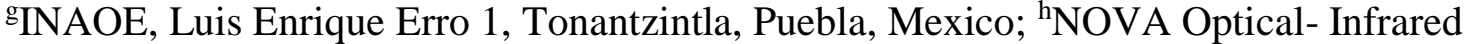 \\ Instrumentation Group at ASTRON, Oude Hoogeveensedijk 4, 7991 PD Dwingeloo, The \\ Netherlands; i Leiden University, Leiden Observatory, P.O. Box 9513, 2300 RA Leiden, The \\ Netherlands.
}

\begin{abstract}
WEAVE is a wide-field spectroscopy facility for WHT which includes a multi-object dual-beam spectrograph which will operate in the visible wavelength range. The blue beam will cover the range 360-600 $\mathrm{nm}$ and the red arm will cover the $580-960 \mathrm{~nm}$ range. In these ranges the spectrograph will offer a mid-resolution $(\sim 5000)$, while in three narrower wavelength intervals, two for the blue arm and one for the red one, the instrument will provide a high ( 20000) spectrograph resolution. The spectrograph is currently entering the assembly and integration phase and the first light is foreseen in 2019. The entire WEAVE project is managed by an international consortium led by the University of Oxford. The spectrograph is controlled by a coordination process, the so called High-Level Server, which is part of the Observatory Control System (OCS) software suite, and is the single point of access to the embedded control system, the so called Low-Level Control Software, which is based on PAC technology.

This paper describes the design of the embedded software for the control of the spectrograph mechanisms.

We first describe the interface between high and low level software, then we present the PAC architecture and discuss the low-level state machine. Finally, we provide details on the principal program routines and describe the engineering interface.
\end{abstract}

Keywords: Instrument Control Software, Programmable Automation Controller, Software Architecture.

\section{INTRODUCTION}

William Herschel Telescope Enhanced Area Velocity Explorer (WEAVE) is a wide-field spectroscopy facility for the WHT $^{1}$ including a multi-object dual-beam spectrograph which will operate in the visible wavelength range $360-960 \mathrm{~nm}$. It is fed from a multi-fiber positioning system (POS), hosted on top of the telescope, where a new two-degree field of view prime focus corrector is also placed. The spectrograph is entering the assembly and integration phase ${ }^{2}$ and the first light is foreseen in early 2019. In this paper we present the architecture of the embedded control software, designed at ING and implemented at INAF-Oapd in collaboration with the IAC. The entire WEAVE project is managed by an international consortium led by the University of Oxford. An overview of the instrument, the mechanisms under software control and of its network architecture is provided first. Then a description of the complexities encountered whilst developing the Low-Level Software (LLS) is provided.

*bernardo.salasnich@inaf.it; phone: +39049 8293494.

Software and Cyberinfrastructure for Astronomy V, edited by Juan C. Guzman, Jorge Ibsen, Proc. of SPIE Vol. 10707, 107071X · (C) 2018 SPIE · CCC code: 0277-786X/18/\$18 · doi: 10.1117/12.2311469 


\section{THE SPECTROGRAPH}

Figure 1 shows a schematic overview of the mechanisms under software control. The light enter the instrument through 1000 fibers to an exchange mechanism, which allows the selection of one of four slit configurations: two for multi object fiber spectroscopy (MOS-A and MOS-B), one using a large single integral field unit (LIFU) and one composed of twenty small multi integral field units (MIFU). The mechanism can move to the position corresponding to the selected configuration through a tip and tilt movement; at that moment the selected slit head can be inserted. The exchanger mechanism is equipped with four back-illumination units, which, when requested by the positioner, can illuminate the slit and allow the fiber gripper unit in the positioner to image the fibers using its dedicated camera. The beam from the fibers is then collimated and reaches a dichroic beam splitter, located after the collimator, which divides the beam into a blue and a red arm. Inside each arm, the light passes through a shutter to a deployable volume phase holographic (VPH) grating, which disperses the light. Depending on the selected resolution, there are two VPHs in the red path and three in the blue. Finally, the beam is collected by a thermally controlled CCD camera. The opening of the CCD shutter (not shown in figure) is not under LLS control but the software can switch on/off its power supply under particular conditions. The blue beam will cover the range 360-600 nm and the red one will cover the 580-960 nm range. In these ranges the spectrograph will offer a mid-resolution ( 5000). Moreover, in three narrower wavelength intervals, two for the blue arm and one in the red one, the instrument will provide a high $(\sim 20000)$ spectrograph resolution. The change between high and low resolution in an arm is operated in a rather spectacular way by first lifting the entire arm with dedicated air-pads, then by rotating the entire structure and finally lowering the arm into position. Then the VPH, corresponding to the given resolution, is inserted. Any change of resolution requires an adjustment of the tilt angle and of the focus position of the whole detector cryostat. A similar floating/move/landing sequence is used to adjust the position of the module. Each arm is then equipped with flat field sources to illuminate the detector.

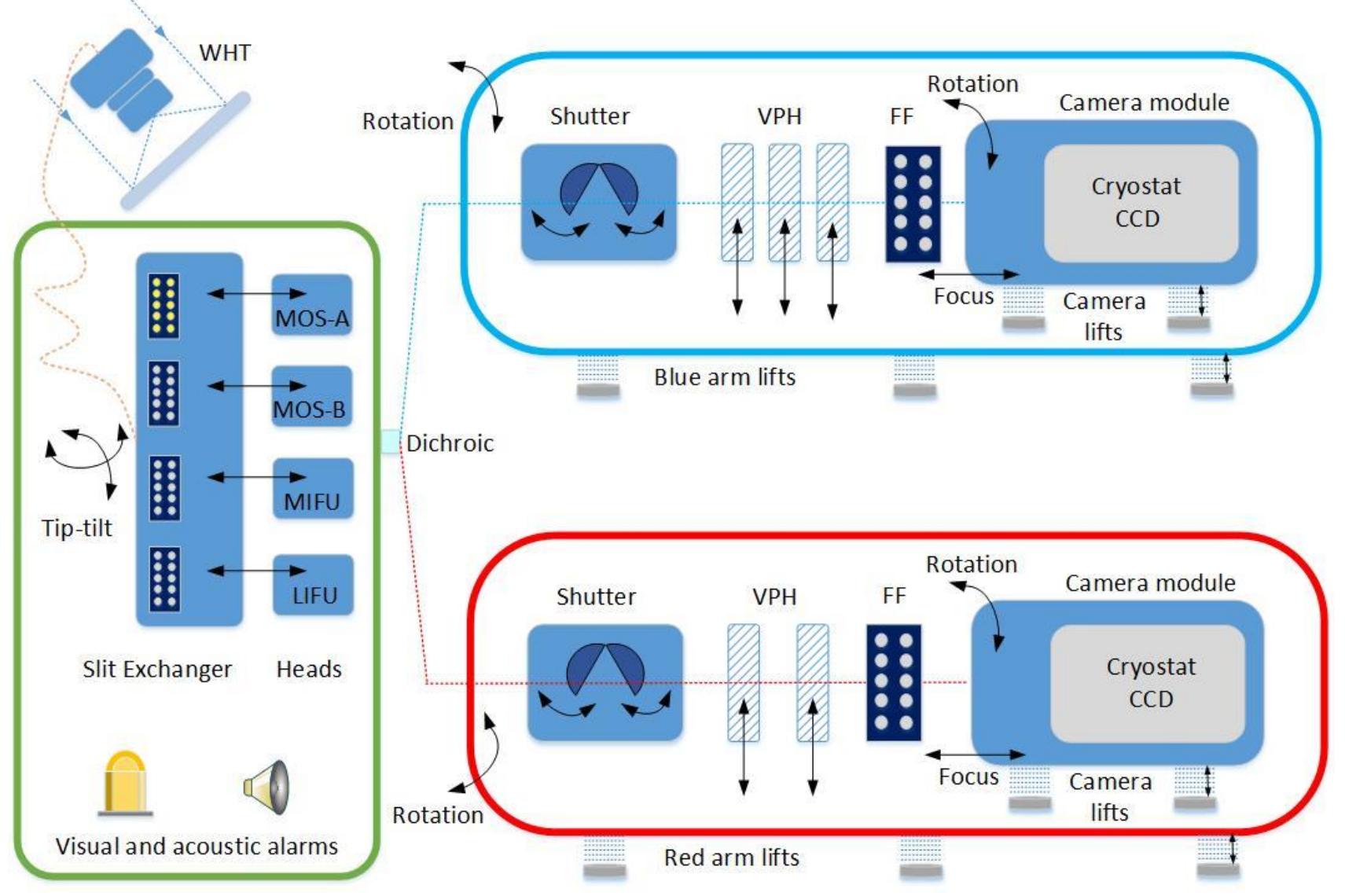

Figure 1. Schematic overview of the spectrograph with the mechanisms under LLS control. 
Figure 2 shows the network architecture of the instrument. The mechanisms are controlled by a Control-Logix 1756-L73 Programmable Automation Controller (PAC). Most terminals are distributed over three electronic boxes, physically separated and located on top of the spectrograph cover, connected via Ethernet by 1756-EN2T adapter modules. This deployment corresponds to the three sub-systems within which the spectrograph can be functionally divided: the common path hosting the fiber exchange mechanism and the two arms. Each module inside each box belongs to the Allen-Bradley 1734 family. Finally, the main chassis hosts a High-Speed Digital Input module to receive from the POS the signal to enable the back-illumination units and a dual port module for serial communication (ProSoft Technology MVU56E family) to interface with the camera tilt and focus mechanisms. The PAC can communicate via Ethernet with each box and it is accessible by the RSLogix Studio 5000 development tool, the Engineering Control Panel, hosted on a dedicated Engineering Interface Server, and by the High-Level Software.

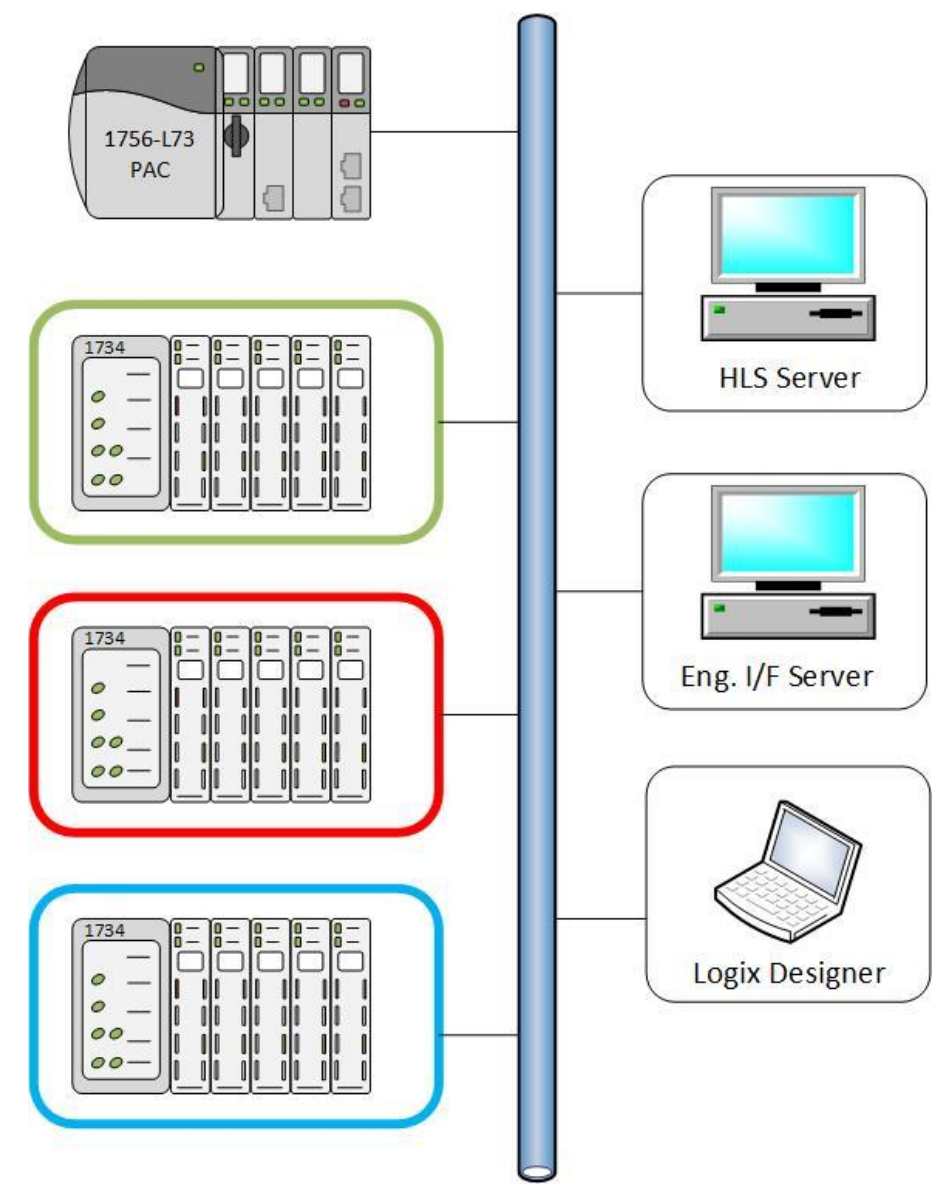

Figure 2. Schematic overview of the network.

\section{THE CONTROL SOFTWARE}

The spectrograph is mainly a pneumatic instrument: 18 of a total of 32 mechanisms under software control are compressed-air driven devices (there are 14 pistons and 4 lifting mechanisms). The four devices responsible for the fine movement of the camera module, for tilt and focus adjustment, are linear actuators driven by DC motors. The 10 internal light sources units for back illumination and flat field are led driven.

The choice of a PAC and of pneumatic actuators comes from the experience acquired on these technologies at the WHT, where other instruments based on them already exist, like the ISIS spectrograph. 


\subsection{Simple section of LLS}

The embedded Low-Level Control Software (LLS) is designed to perform only simple and atomic operations on each device. It means that complex movements, as sequences of movements or 'recipes', are reserved for the High-Level Control Software (HLS), which is part of the Observatory Control System (OCS) software suite ${ }^{3}$. From the point of view of the HLS and the Engineering Control Panel application, the spectrograph is a black box controllable through a very simple interface: four global variables, or tags, are published in the PAC memory where they can be accessed by HLS using the standard PLCIO library. An index is reserved to specify which mechanism to control, an index to specify the command to be executed on the selected mechanism, a floating point number to give an optional parameter to the command, corresponding most of the times to the demanded position, and a logical flag to trigger the execution of the command. It is responsibility of LLS to check for the validity of the received commands and to activate the corresponding functions.

Each PAC is designed to execute the programmed instructions in a continuously running loop, called the Scan Task, which takes $\sim 5 \mathrm{~ms}$ to complete. No complete control operation can be performed over a scan period. The philosophy is to activate a function which manages an internal tag. This tag is called the phase and is set to zero when the mechanism is idle. For example, suppose the spectrograph is in maintenance mode. When the function is called to move a mechanism, it activates the visual and acoustic alarms as a warning that the maintenance mode is active and starts a 5-s timer. At this stage, the phase is still zero. After 1000 scans (which corresponds to 5s) the timer elapses and the phase is set to 1 . In the case of a piston, the alarms are then switched off, the PAC reads the current piston position and sets the phase to 2. If the piston position is at the demanded position, there is no need to continue and control passes to the final phase, in this example, phase 100. If the piston position is different from the demanded position, the output signal is set to a value which corresponds to the piston solenoid activation signal and the phase is set to 20. At phase 20, the routine reads the piston position and will not change the phase tag until the end of the movement, at which stage the phase is set to 100 , unless there is a timeout. At phase 100, the 'piston is busy' flag is reset and the phase is set to 0 thus allowing the piston to receive a further command.

The adopted programming language is Structured Text, only the main routine is written in Ladder Diagram.

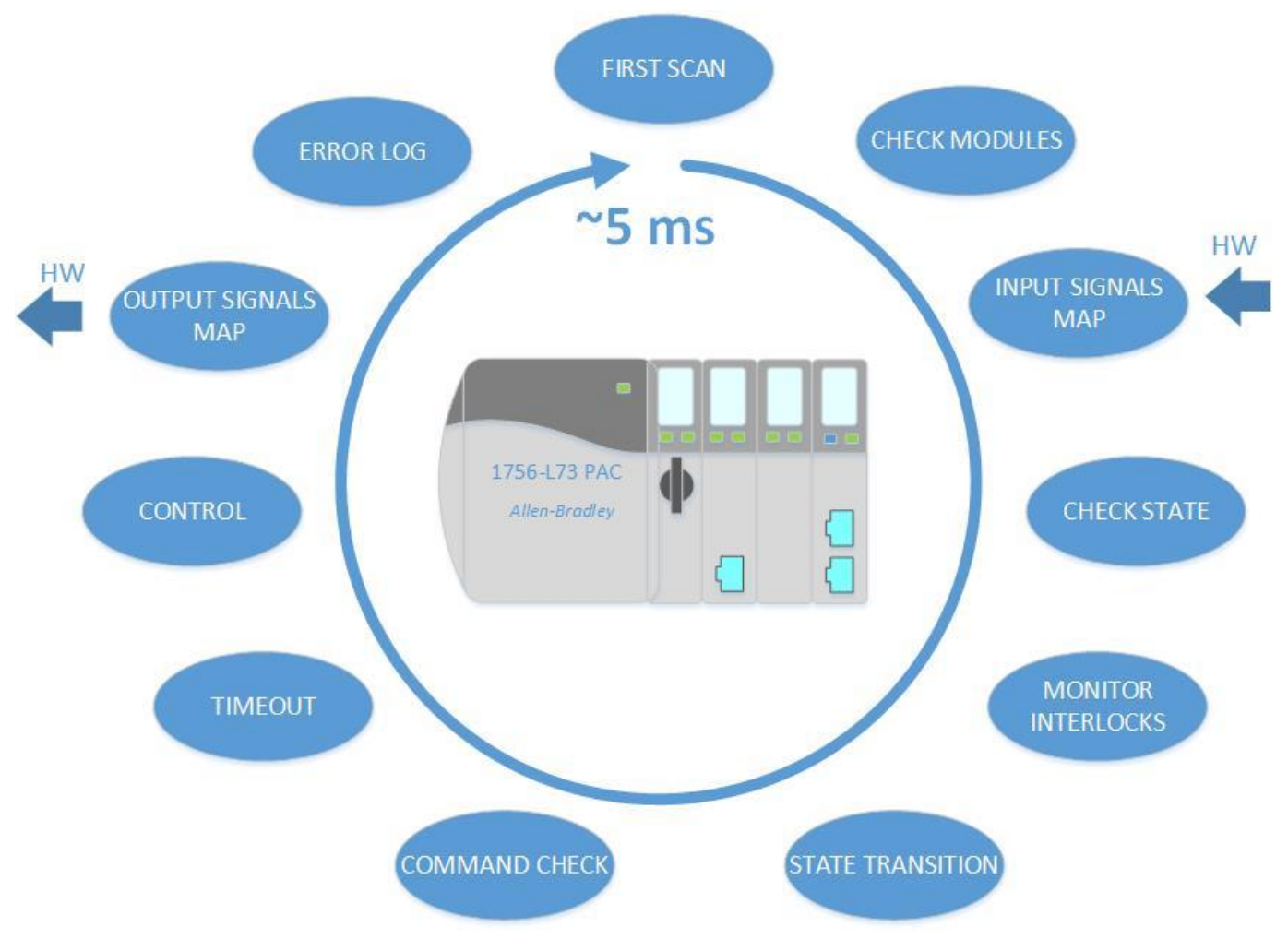

Figure 3.Sequence of routines called during the main task 
Figure 3 shows a simplified diagram of the routines called during each scan cycle. They are briefly described below:

- The FirstScan routine is called once the PAC is booted. It is used to initialize the internal tags.

- The CheckModules routine is responsible for checking the Allen-Bradley modules in order to detect any communication failures.

- InputSignalMap is called to take a snapshot of the input signals (digital and analog) and map them into internal tags.

- CheckState verifies the current position of all mechanisms, checks that they are at known positions and if the current position combination is allowed.

- For each device the MonitorInterlocks routine verifies that the mechanism is not interlocked by other mechanisms or by failure conditions, due to, for example, the air pressure being below a configurable threshold or to an unauthorized opening of the instrument cover.

- The routine StateTransition handles the instrument state machine and performs the state transitions.

- The CommandCheck routine verifies that the command received from the HLS (or from the Engineering Panel application) can be executed, by checking the parameters, the current state machine, the state (idle or busy) of the selected mechanism and by rejecting the command if any failure has been detected.

- The Timeout routine is responsible for handling the timers associated with each mechanism. For example a fault condition is raised if the movement exceeds a configured time threshold. Moreover, this routine handles some auxiliary timers, used for example to introduce a small delay at the transition from NOT_INIT to RESTART allowing the air relays to operate and remove the air.

- The Control routine manages the actions to be performed on the mechanisms and it is split, for convenience, into three routines: one to control the pistons, one for the motors and one for the light sources. Each mechanism structure contains a dedicated tag where the current execution phase is stored, as explained before. A mechanism cannot receive a new command if its phase is not zero.

- Each control sequence ends by resetting the phase and by setting the values of the output signals, which are mapped to the physical outputs in the OutputSignalMap routine.

- Any failure is then caught and stored in a dedicated buffer by the ErrorLog routine. The buffer is then read and displayed by the Engineering Control Panel application.

\subsection{Complex section of LLS}

The LLS complexity resides in the handling of the mechanism interlocks and in the state machine design, where special attention has been paid to the restarting procedure after an emergency episode or a failure.

Mechanism interlocks are monitored at each scan by a dedicated routine. A simple example of this is the case when the camera rotation mechanism is not at the low resolution position; any movement of the low resolution grating in that arm is prevented because it could damage the VPH. More than one hundred combinations are monitored by the LLS.

The restarting procedure is handled exploiting a state machine, shown in Figure 4.

The spectrograph has six states:

1. NOT-INIT: in this state, the air relays are deactivated. No air is present in the tubes, all light sources are switched off, the CCD shutters are powered off, the acoustic alarm is not buzzing and the visual alarm, unless the maintenance key is enabled, is off. It is not possible to move any device and all commands are rejected. The operator is informed that it is possible to carry out a RESTART by pressing a dedicated button which starts blinking.

2. RESTART: in this state, the air relays are activated, the CCD shutters are powered on, the acoustic alarm is not buzzing and the visual alarm, unless the maintenance key is enabled, is off. The Restart button is switched on.

3. NORMAL: in this state, the instrument is fully operative, mechanisms can be moved in parallel, acoustic and visual alarms are off.

4. MAINTENANCE: in this state, the maintenance key is enabled and the instrument is operative, but mechanisms cannot be moved in parallel. A command to a mechanism is rejected if another one is moving. The operator is allowed to open the instrument cover. For this reason, a particular acoustic alarm is enabled for a few seconds before any movement and a steady visual alarm is activated. Both CCD shutters are powered off.

5. SHUTTER: this state is designed to allow the maintenance of the CCD shutters, which are both powered on. The shutter maintenance key is enabled and it is not possible to move any mechanism.

6. EMERGENCY: in this state, the compressed air is removed from the whole system with the exception of the air-pads, which are enabled to allow the spectrograph arms to float. This allows the operator to manually move 
them if needed. The CCD shutters are powered off, the acoustic alarm is on and the visual alarm is on and blinking. It is not possible to move any device, all commands are rejected and a description of the emergency is clearly visible in the Engineering Control Panel.

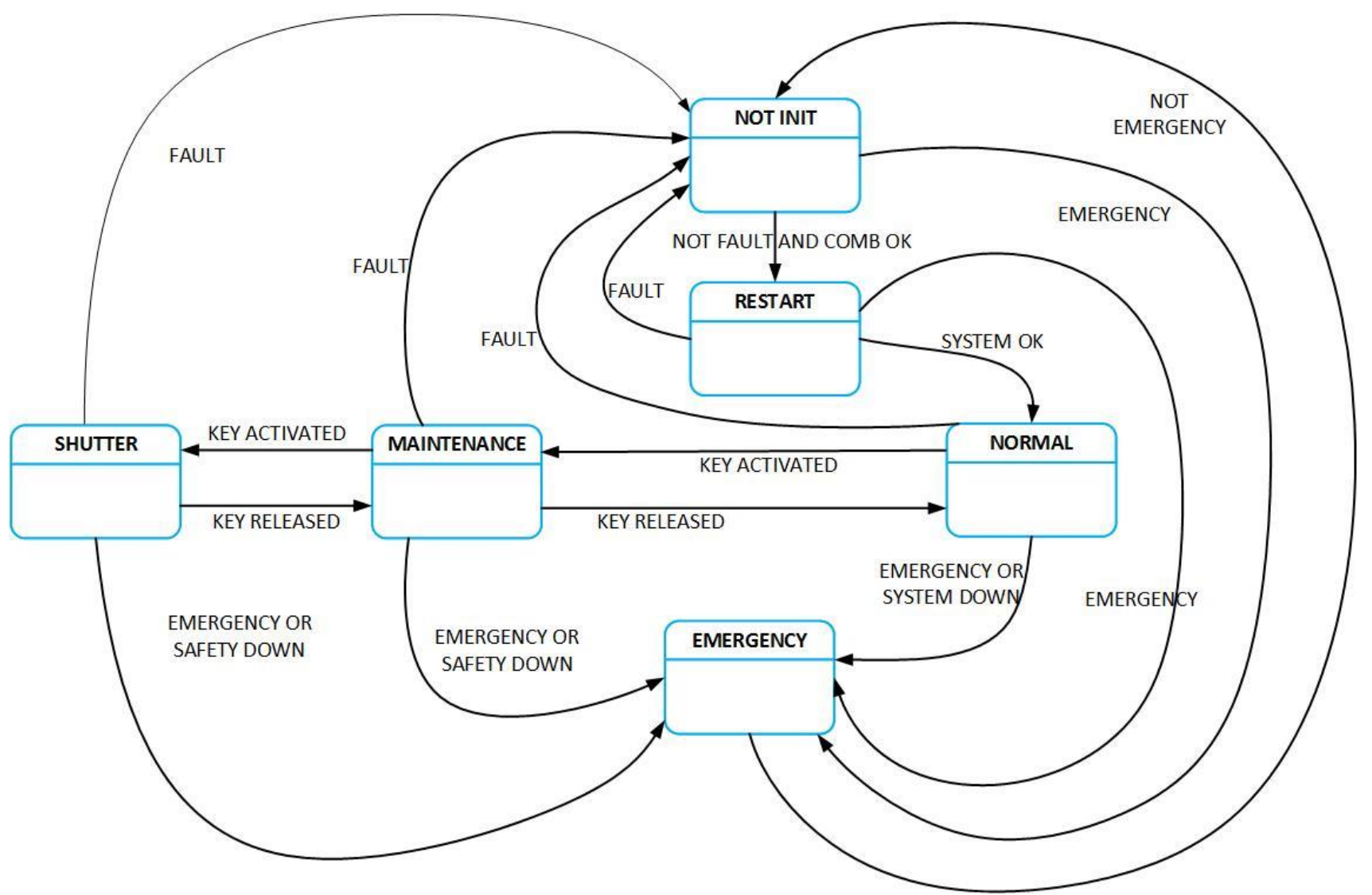

Figure 4. State machine diagram. Six states are foreseen. Transitions between them depend on the logical combinations of physical quantities (air-pressure, voltages) monitored at each scan cycle and statuses of two maintenance keys.

During each state transition, the following conditions are checked:

- FAULT means that at least one of the following conditions occurred:

$\circ$ the system detected that a piston is at an unknown position, which means that for some reason it stopped in the middle of its motion range;

0 there is a failure in the main air system (the pressure is out of range);

$\circ \quad$ there is a fault in the communication between the PAC and an electronic box;

$\circ$ there is a failure with the power supply of an electronic unit;

$\circ$ a timeout, for example when a mechanism takes too long to move to the demanded position. Each device has its own configurable timer.

- EMERGENCY means that an emergency push button has been pressed by an operator or one of the seven instrument covers has been removed without first activating the maintenance key;

- COMB_OK means that all pistons are at a known position and that the current combination of positions is allowed. This is extremely important in order to avoid any damage to the system when the air valve is released and a pressure of almost six atmospheres flows into the system. For example when the system is in EMERGENCY state, without air in the tubes, the operator could open the instrument cover and manually move the Slit Exchange device to a LIFU configuration and insert a MOS slit head. This combination is not allowed and it is detected by the LLS, which prevents the system from passing to the RESTART state and keep deactivated the air-valve. 
- SYSTEM OK/DOWN provides an additional security level by reading the status of a dedicated safety hardware valve.

- KEY status: the electronic box controlling the common path devices is equipped with two keys which allows the maintenance mode and the CCD shutter maintenance mode to be enabled or disabled.

\subsection{Engineering interface}

Figure 5 shows the Spectrograph Status Panel, which was developed using the RSView32 tool by Allen-Bradley. The information displayed on the right side of the panel is grouped by electronic units, SEBOX, REDBOX and BLUBOX corresponding respectively to the common path, red arm and blue arm sub-systems. The columns illustrate:

- the status of the Ethernet communication (failure, normal);

- the status of the power supply units (on, off);

- if an electronic box is available (a temporary feature added for the integration phase);

- if the mechanisms of the given sub-system are at a known position;

- if the current combination of positions is allowed;

- the status of each instrument cover (open, closed);

- the status of the emergency push buttons (pressed, released); and

- the air pressure on each sub-system.

The general state of the instrument is displayed at the top, together with the main air-supply status. The status of the maintenance keys is shown on the bottom, together with the status of the CCD shutter power supplies. The control software is continuously monitoring the state of an external device used to refill the cryostat tanks. When the operator starts the filling operation of one tank, the LLS interlocks the rotation of the arm on which the tank is mounted and displays it in the panel through a blinking LED.

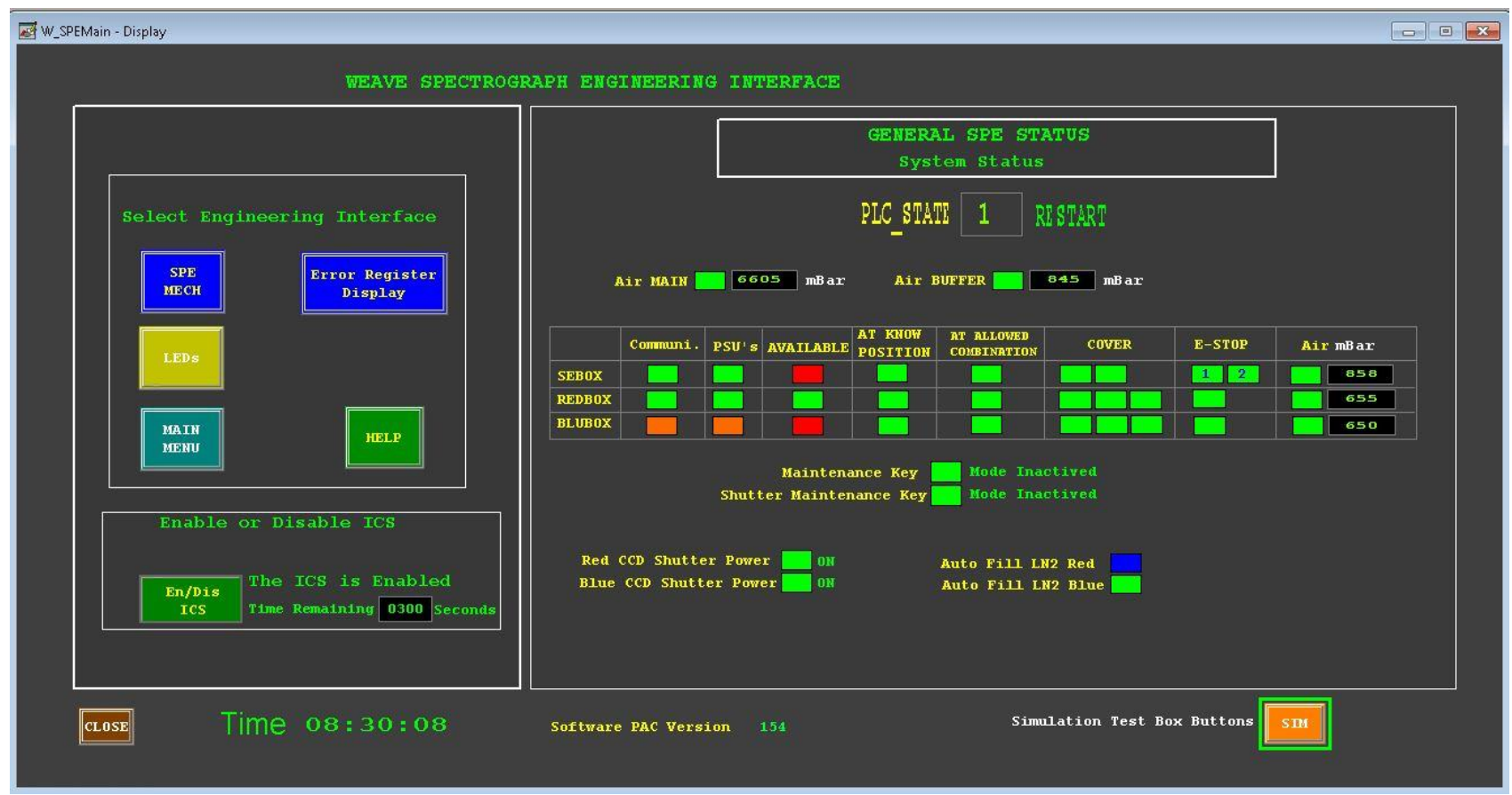

Figure 5. Spectrograph Engineering Status Panel. See text for the details.

On the left side the user can launch other engineering interfaces, not presented in this paper. One is aimed to control the back illumination and flat field sources, and it is launched through the "LEDs" button. A second panel is used to list the 
last one hundred error messages and it is launched through the "Error Register Display" button. The "MAIN MENU" button offers a short summary of the spectrograph status. Moreover a dialog is accessible via the HELP button to describe the panel entries. On the bottom left side the telescope operator, for maintenance purposes, can isolate the LLS from the HLS through the "En/Dis ICS" button. In this case any command received from the HLS is rejected for a configurable amount of time. A counter shows the elapsed time.

Finally, "SPE MECH" button allows the system to switch to the Engineering Control Panel, shown in Figure 6.

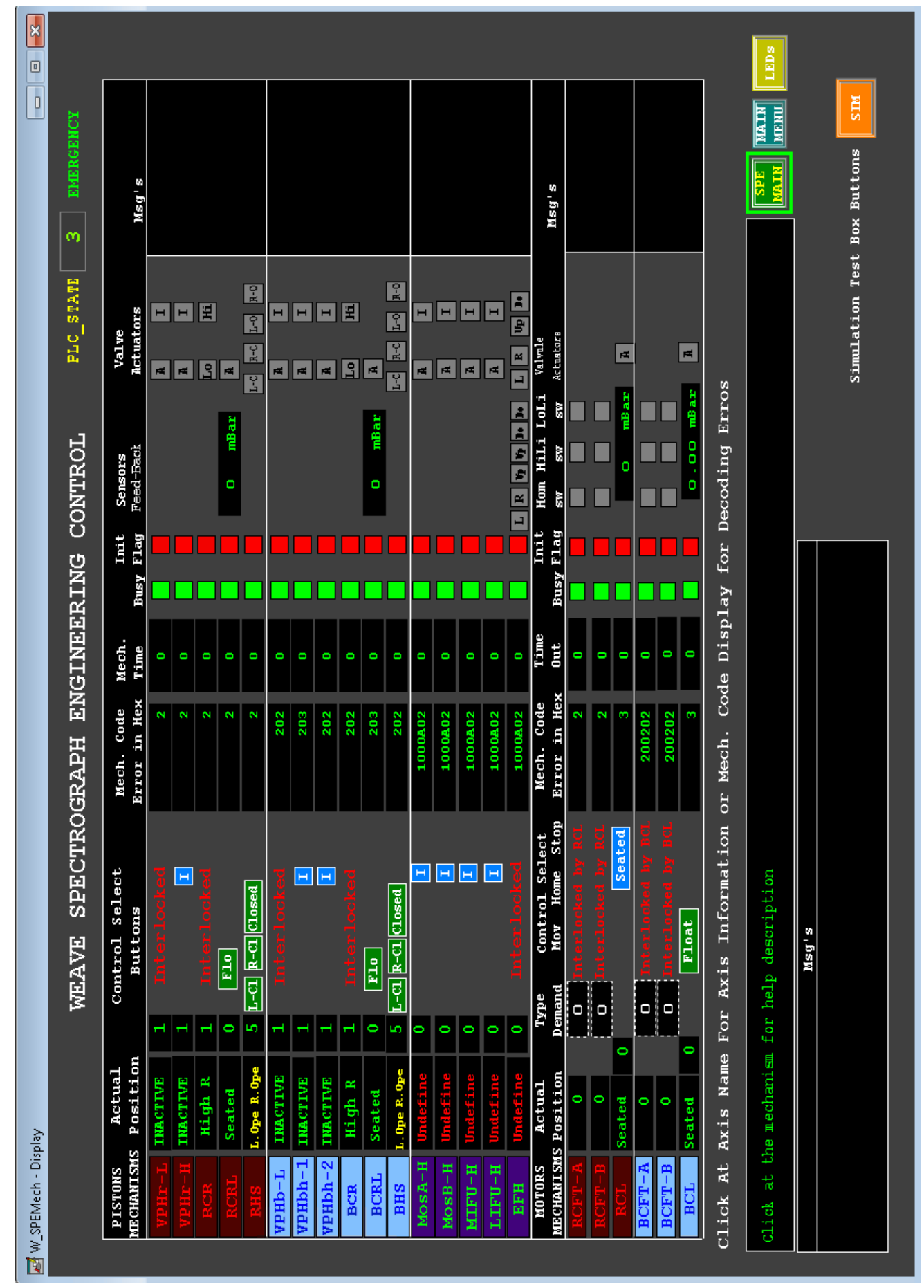

Figure 6. Spectrograph Engineering Control Panel. See text for the description. 
From the Engineering Control Panel all pistons and motors can be controlled. The panel is divided into two frames, the upper frame dedicated to control of the pistons, sorted by REDBOX, BLUBOX and SEBOX. The bottom frame is reserved to the motors, for control of the tilt and focus adjustment of the detector module.

For each mechanism, the panel displays by column:

- the actual position;

- one or more buttons -depending on the mechanism- to move the device to an allowed position;

- the error code;

- the duration of the motion;

- the status of the valve actuators (active or inactive).

The last column is used to display additional information about particular events which can occur on each mechanism, like for example "arm rotation interlocked by liquid $\mathrm{N}_{2}$ filling".

\section{REFERENCES}

[1] Dalton, G.B. et al, "Construction progress of WEAVE: the next generation wide-field spectroscopy facility for the William Herschel telescope", paper 10702-47, this conference.

[2] Stuik, R. et al, "Integration and testing of the WEAVE spectrograph", paper 10702-275, this conference.

[3] Picó, S. et al, "The WEAVE observatory control system", paper 10704-83, this conference. 